\title{
Effect of web-based tailored lifestyle interventions on fruit and vegetable consumption in adults: A systematic review and meta-analysis of randomised controlled trials
}

\author{
C. Celis-Morales, S. Abraham, P. Keenan, A. W. Ashor, K.M. Livingstone, J. Lara \\ and J. C. Mathers \\ Human Nutrition Research Centre, Institute for Ageing and Health, Newcastle University, Campus for Ageing and \\ Vitality, Newcastle on Tyne, NE4 5PL, UK
}

Although the health benefits of greater fruit and vegetable (FV) consumption are well known ${ }^{(1)}$, a large proportion of the UK population fails to meet the recommended intake of at least 5 portions per day ${ }^{(2)}$. Personalised (or tailored), web-based nutritional interventions are becoming a popular strategy for improving FV intake but the effectiveness of such web-based interventions in increasing FV intake remains unclear. The objective of the present study was to conduct a systematic review and meta-analysis of randomised controlled trials (RCTs) which tested the effect of personalised, web-based nutritional interventions on FV intake.

Seven databases (ASSIA, CAB Abstracts, IBSS, Medline, Psych Info, Scopus and Embase) were searched using the following criteria: 1) RCTs; 2) tailored versus non-tailored advice; 3) web-based interventions; 4) dietary-related outcomes; 5) adult participants $\geq 18$ years. Data were pooled as weighted mean difference (WMD) and analysed using a random effects model.

Nine studies were conducted in United States and the remaining studies in the Netherlands, Australia and Belgium. The mean age of participants in the studies ranged from 27.4-61.2 years. Pooled analysis of 13 studies $(n=5,465$ participants $)$ showed that webbased personalised interventions were more effective in increasing FV consumption (Fig 1. WMD: 0.41 serving portion per day [95\% CI: 0.22 to 0.61$] ; p<0.0001)$ than non-personalised advice.

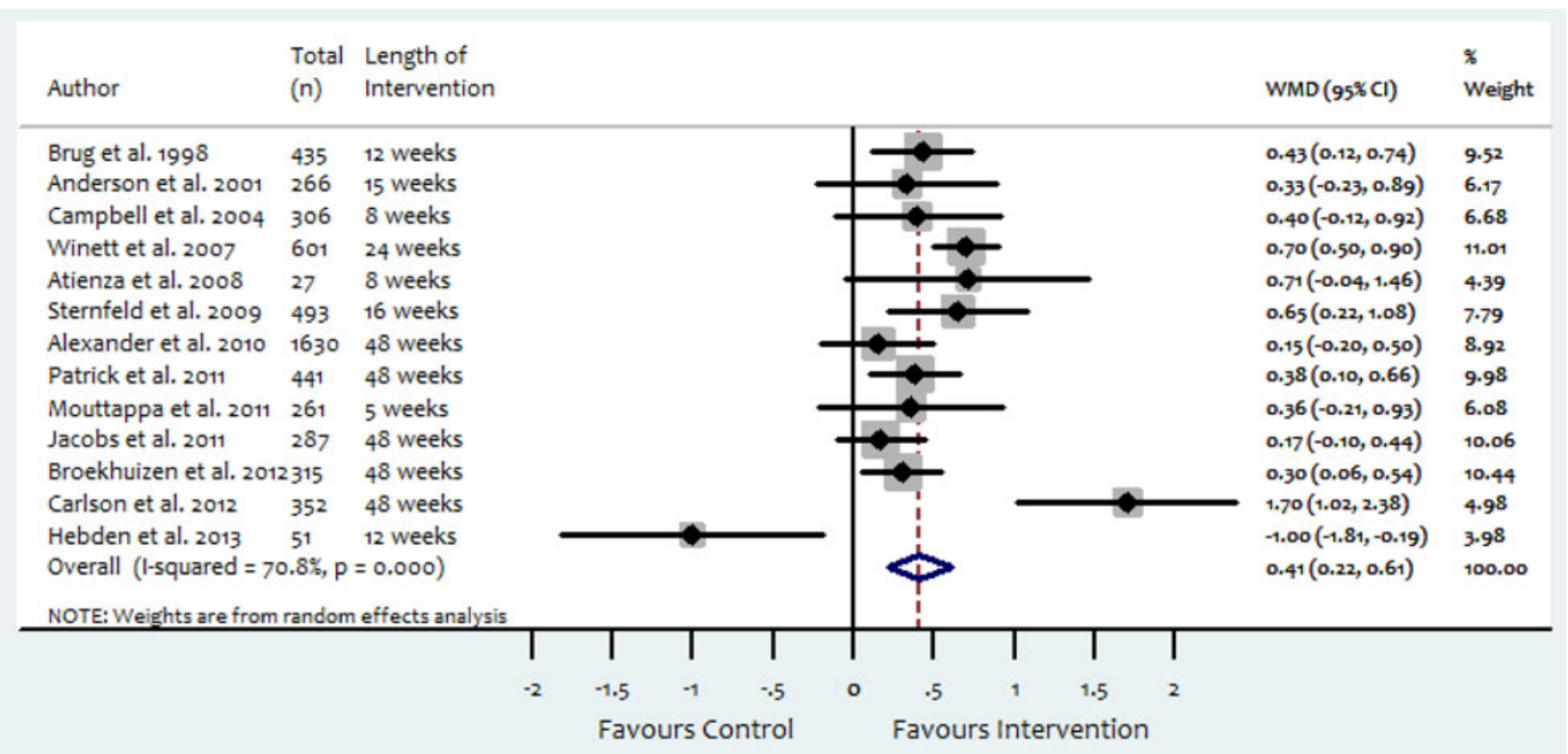

Fig. 1. Forest-plot of randomised clinical trials investigating the effect of web-based personalised interventions on fruit and vegetables consumption (data expressed as serving portions per day).

These results suggest that the use of web-based, personalised interventions could be a more effective strategy for increasing FV consumption in the adult population, than non-personalised advice.

1. Leenders M, Sluijs I, Ros MM, et al. (2013) Am J Epidemiol 178, 590-602.

2. NHS. (2013) http://www.nhs.uk/livewell/5aday/pages/5adayhome.aspx 